\title{
In Situ Observations of Post-Synthesis Modification of a Metal-Organic Framework Using Atomic Resolution S/TEM and EELS
}

Peter Tieu ${ }^{1}$, Chaitanya Gadre ${ }^{1}$, Wenpei Gao ${ }^{1}$, Xingxu Yan ${ }^{1}$, Djawhar Ferrah ${ }^{1}$, Muqing Li $^{2}$, Zhengtao Xu ${ }^{2}$ and Xiaoqing $\operatorname{Pan}^{1}$

${ }^{1}$ University of California - Irvine, Irvine, California, United States, ${ }^{6}$ City University of Hong Kong, Kowloon Tong, Hong Kong

Post-synthesis modifications (PSMs) of metal-organic frameworks allow for effective enhancement of the reactivity and properties of the base structure. [1] Much work has been done investigating additions to the framework or metal center. [2] However, elucidating the structural changes remains a challenging task, especially for in situ thermal PSM which tends to remove material from the host net. Therefore, it is crucial to be able to observe the structure directly and examine when bonding changes occur so as to carefully control the end product. Here, we report the combination of in situ scanning/transmission electron microscopy (S/TEM) with vibrational electron energy loss spectroscopy (EELS) to directly observe lattice contractions and the corresponding changes to the bonds on the organic linker during a thermal PSM. In situ imaging and analysis of the fast Fourier transform indicate a two stage change, disulfide formation and decarboxylation, which directly influence the lattice spacing. Analysis of ex situ samples via STEM imaging, FTIR, and X-ray photoelectron spectroscopy (XPS) validate the chemical bond changes observed in situ.

In situ imaging was performed on a JEOL JEM-ARM300CF with double aberration correction while heating from room temperature to $800{ }^{\circ} \mathrm{C}$ and showed the MOF lattice shrinking. Fast Fourier transformation (FFT) of the observable lattice followed by line profile measurements indicated an initial distance of $2.11 \mathrm{~nm}$ at $200{ }^{\circ} \mathrm{C}$ between lattice points which corresponds to the distance between $\mathrm{Zr}$ clusters. Heating of the sample showed a marked increase in the reciprocal lattice spacing in the FFT between 250 and $300{ }^{\circ} \mathrm{C}$ and again between 500 and $600{ }^{\circ} \mathrm{C}$ which indicates a contraction in real space. The negative thermal expansion (NTE) behavior exhibited by this MOF can be partially attributed to low energy vibrational modes associated with translational movement perpendicular to the benzene ring plane. [3] However, the measured linear coefficient of thermal expansion from 200 to $300{ }^{\circ} \mathrm{C}$ and 460 to $500{ }^{\circ} \mathrm{C}$, $\alpha=-31.8 \times 10-5 \mathrm{~K}^{-1}$ and $\alpha=-13.7 \times 10-5 \mathrm{~K}^{-1}$, respectively, is an order of magnitude larger than similar structures. Additionally, at higher temperatures, a decarboxylation process is known to commonly occur. The premise of NTE with regards to vibration modes requires a relatively flexible bond such as found in the carboxyl group. Therefore, the NTE behavior observed here must be explained by other mechanisms such as bond cleavage and formation. To study the changes which involve breaking and reforming bonds, vibrational EELS was selected to complement the imaging.

To investigate the formation of new bonds, vibrational EELS was utilized while heating the MOF structure in a Nion HERMES UltraSTEM 200 at $60 \mathrm{kV}$. Raman and FTIR data provide the energy of the bonds present in the pristine MOF structure to correlate with the observed peaks in vibrational EELS (Figure 1a). The large, broad peak from $115-225 \mathrm{meV}$ is attributed to the $\mathrm{C}-\mathrm{C}$ and $\mathrm{C}-\mathrm{O}$ bonds while the smaller peak from 60-110 meV is the mixture of C-S and S-S bonds (Figure 1b). A comparison of the peaks at each temperature provides an understanding of the relative ratio between the kinds of bonds present. By accounting for the zero loss peak broadening throughout the experiment, the normalized peak area of the $\mathrm{C}-\mathrm{S}$ and S-S peak was plotted versus temperature and shows an increase at $300{ }^{\circ} \mathrm{C}$ and $500{ }^{\circ} \mathrm{C}$ which 
matches the two stages of lattice contraction observed in the direct images (Figure 1c). This increase in the ratio is indicative of an increase in the number of bonds present which is from the formation of the disulfide bond at $\sim 300{ }^{\circ} \mathrm{C}$ and the decarboxylation at $500^{\circ} \mathrm{C}$. The formation and cleavage of these bonds between adjacent organic linkers pulls the zirconium clusters closer to each other which results in the shrinkage of the lattice.

Correlative ex situ measurements were performed to corroborate the vibrational EELS results. XPS was used to identify the nature of the chemical bonding in the MOF structure. C1s and S2p core levels were collected before and after annealing samples to varying temperatures to determine the probable structures (Figure 2a-b). Annealing to $500{ }^{\circ} \mathrm{C}$ shows the complete removal of oxygen functional groups associated with the decarboxylation process which was also observed with vibrational EELS. The spin-orbit pair for the S2p in the pristine sample show the single chemical environment of the thiol whereas upon heating, additional products associated with phenyl sulfide and phenyl disulfide and oxygenated structures appear (Figure 2c). The formation of the disulfide bond as indicated by XPS match well with the vibrational EELS results observed in situ, indicating the changing bonding nature. From the in situ and ex situ results, we are able to elucidate the likely structural changes during the heating process.

Tracking the formation of a chemical bond in situ provides insight into the reconfiguration process. Unlike with other traditional spectroscopic techniques such as FTIR or Raman, the strength of in situ imaging and vibrational EELS lies in the ability to track structural changes simultaneously with bond changes rather than taking snapshots of the process before and after a reaction. Here, we observed lattice shrinkage in real space during a heating process due to the cleavage and formation of bonds which was explained with in situ vibrational EELS. By understanding the stepwise changes during structural modification, fine tuning of the post-synthesis modification process can be achieved for such applications as controlling pore sizes. Because of the periodicity of a MOF structure, a change in bonds within the organic linker can ripple throughout the entire material which we are able to observe. [4]
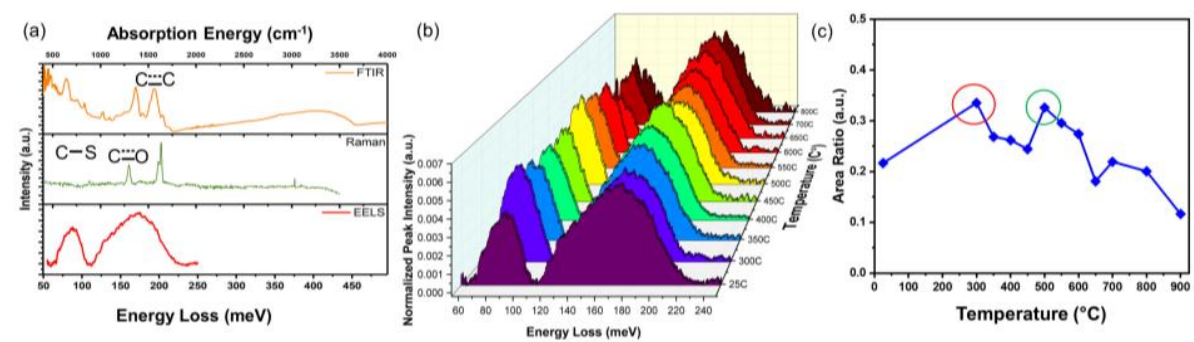

Figure 1. (a) Comparison of peak energies in FTIR, Raman, and vibrational EELS of the pristine MOF structure. (b) In situ vibrational EELS at different temperature observing changes to bonds present. (c) Normalized area of the C-S and C-C peak from (b) showing the increase in relative intensity at 300 (red circle) and $500{ }^{\circ} \mathrm{C}$ (green circle). 

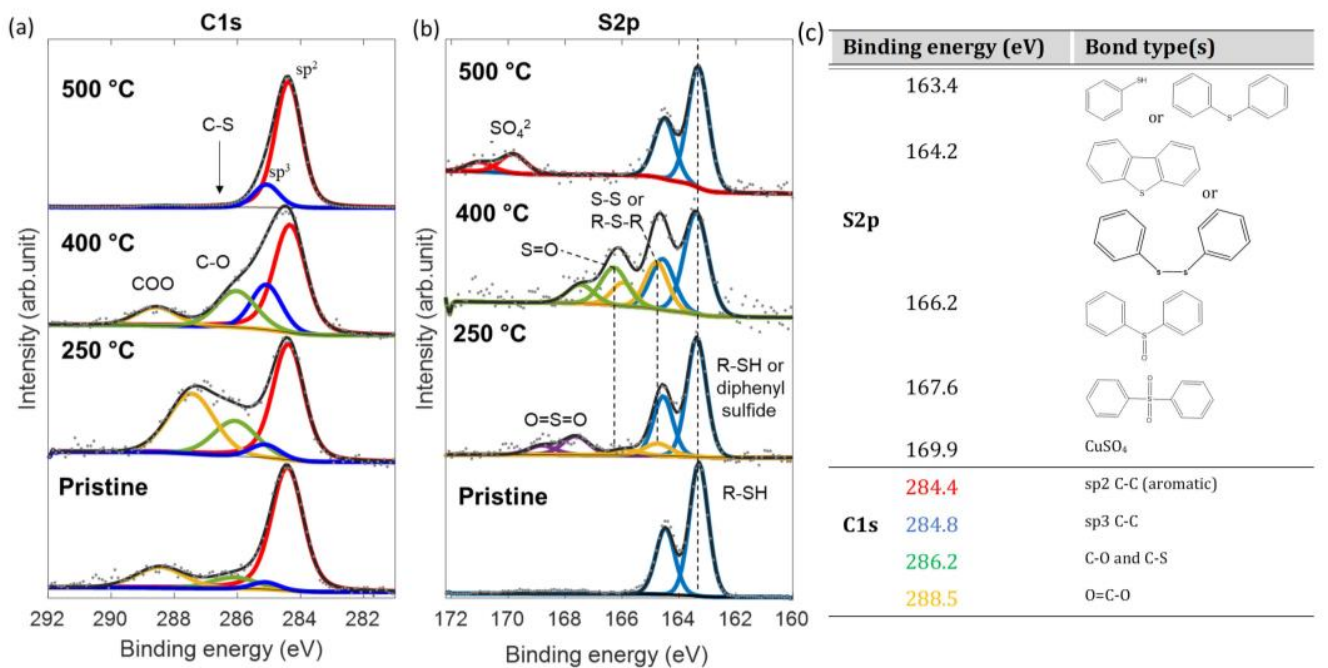

Figure 2. (a) Carbon 1s and (b) sulfur 2p XPS components of ex situ MOF samples at various temperatures indicating the changing sulfur environment and the different bonds breaking and forming. Samples were taken on copper foil. (c) Binding energies and the corresponding structures.

References

[1] Chen, B, et al. J. Am. Chem. Soc. 2000, 122 (46), 11559-11560

[2] Reimer, N., et al. CrystEngComm. 2012, 14 (12) 4096-4104

[3] Zhou, W., et al. Phys. Rev. B. 2008, 78 (5), 1-5

[4] The authors would like to acknowledge Muqing Li and Zhengtao Xu for providing the MOF sample and the corresponding Raman and FTIR data. Images and EELS data were acquired at the Irvine Materials Research Institute (IMRI). 